As mentioned in our paper, we agree with Dr Donnelly about the need for members of the mental health team to work closely with the GP in the management of such patients. However, this collaboration can take various forms, which have yet to be evaluated.

Warneford Hospital

Oxford OX3 $7 J X$

Jose Catalan DENNIS GATH

\section{P3 and CT Scan in Patients with Chronic Schizophrenia}

SIR: We read with interest the comments of Ebmeier et al (Journal, February 1988, 152, 290-291). It is suggested that the increase in P3 latency reported in some studies of schizophrenic patients may be related to treatment with anticholinergics. In our study (Barrett et al, Journal, March 1986, 148, 414 420) we did not find a difference in P3 latency between schizophrenics and normals, but employed a four-way tone discrimination paradigm with a longer inter-stimulus interval than is usual (1.8 s). We compared patients on anticholinergics with a group on neuroleptics alone and found no significant difference in P3 latency. However, N1 latency was significantly longer in the anticholinergic group.

We would agree that it is a selected group of patients who agree and are able to participate in this type of psychophysiological study. The more emotionally blunted or thought disordered patient would not, in our experience, be able, willing, or interested in performing the tasks involved. Hostile and suspicious patients steer clear. Our group was predominantly 'paranoid' (on RDC subtyping; $n=$ 16/20). The small non-paranoid group did in fact have significantly longer $P 3$ latency than the paranoid group (P3 latency at PZ in ERPs to infrequent 'target' stimuli: paranoid group $=353 \pm 41$; nonparanoid group $=379 \pm 41$ ). It would be interesting to know if P3 latency in schizophrenics bears any relationship to the cerebral atrophy in this group.

Finally, there is good evidence that the cognitive variables that influence the various ERP components do not do so by raising or lowering a single 'wave', but have rather more prolonged effects which overlie one or more components. We found that a mean amplitude measure from 276 to $426 \mathrm{~ms}$ was more discriminating between normals and schizophrenics than measures of amplitude or latency. These differences were more marked in ERPs to frequent 'nontarget' stimuli than in ERPs to infrequent 'target' stimuli. Subsequent work has indicated that prominent late positivity to non-targets only occurs where longer interstimulus intervals and more complex tasks are involved.

\section{Department of Postgraduate Medicine \\ University of Keele \\ Thornburrow Drive \\ Hartshill, Stoke-on-Trent}

K. BARRETT

W. C. McCallum

P. V. Pocock

Burden Neurological Institute, Bristol

\section{Unilateral auditory hallucinations}

SIR: Khan et al (Journal, February 1988, 152, 297 298) report unilateral auditory hallucinations arising from left otitis media in a chronic schizophrenic patient. They did not locate any of the many similar reports or my extensive but not exhaustive review (Gordon, 1987). I will list some of its conclusions in the light of more papers I have since unearthed.

(a) Hallucinations in various sense modalities can arise from the ear or labyrinth (Ireland, 1893), although only auditory ones will be considered here.

(b) Otopathic hallucinations arise more from otitis media (often serous) than from cochlear deafness. Robinson (1927) found middle ear deafness in $61 \%$ of hallucinating mental patients, compared with $21 \%$ of the non-hallucinating, whereas the inner ear figures were $22 \%$ and $23 \%$.

(c) Ear disease is very common in the insane. Robinson found normal ears and hearing in only $14 \%$ of the hallucinating and $56 \%$ of the non-hallucinating patients, and that was without audiometry or tympanometry. At the turn of the century Fraser found chronic otitis in 5 out of 22 hallucinating patients (Henderson et al, 1913), but abandoned further attempts to establish a pathological basis for tinnitus since they were untestable.

(d) The question of lateral bias is intriguing. In this Journal in 1901 Robertson reported a marked sinistral bias (Gordon, 1987), but Robinson (1927) found a dextral preponderance of ear disease (10 right, 2 left).

(e) Tinnitus is probably a necessary condition for production of hallucinations. Over half with definite hallucinations complained of tinnitus, and most of the rest had ear conditions favouring its occurrence (Robinson, 1927).

(f) The crucial question is whether tinnitus is also a sufficient condition. Robinson thought not. However, Bjeljakow (Ireland, 1893) thought ear infections could lead to insanity and even secondary dementia (i.e. schizophrenia). Peripheral irritation 
could cause heightened cortical excitability and hence unilateral hallucinations.

(g) Hallucinations can sometimes lateralise to the good ear (Robinson, 1927). Electrical aural stimulation in cases of hallucinations and ear disease paradoxically affected the opposite ear (Ireland, 1893). In McCay \& Healey's (1931) case a right radical mastoidectomy cured long-standing mental disorder including sinistral hallucinations. No brain disease was detected. A similar case of 'toxic insanity' came to post-mortem (Henderson et al, 1913). A man became very confused and irritable with hallucinations during an acute exacerbation of chronic otitis. There was considerable improvement of his mental condition with successful ear treatment, but he relapsed and died. There were old and new labyrinthine bone fistulas, but the brain was remarkably healthy.

(h) 'Temporal lobe' epilepsy can also arise from the ear (Ireland, 1893).

(i) Hallucinations can be attenuated by plugging the ear or induced by removing impacted wax (Ireland, 1893).

(j) Peripheral audiosensitivity can lead into hallucinations (Ireland, 1893).

\section{Love Walk}

A. G. GordoN

London SE5 8AD

\section{References}

GoRDon, A. G. (1987) Otopathic hallucinations. Acta Psychiatrica Scandinavica, 75, 664-668.

Henderson, D. K., Mutrhead, W. \& Fraser, J. S. (1913) A case of toxic exhaustive insanity associated with chronic suppurative otitis media, labyrinthitis, and extra-dural abscess. Review of Neurology and Psychiatry, 11, 565-576.

IRELAND, W. W. (1893) German retrospect. Journal of Mental Disease, 39, 299-302.

MCCAY, A. \& Healey, F. H. (1931) Aural sepsis in relation to mental disorder. Journal of Mental Disease, 77, 193-195.

RoBnson, G. W. (1927) Aural disease in the insane. Journal of Neurology and Psychopathology, 7, 332-337.

SIR: We were interested to read the letter of Khan et al (Journal, February 1988, 152, 297-298) concerning a patient with unilateral complex auditory hallucinations. We report two further cases.

Case Reports: (i) A 58-year-old man was readmitted with his third episode of psychotic depression, characterised by depressed mood, loss of drive, grossly disturbed sleep, and loss of appetite and weight. Mental state examination revealed complaints of "a whistling noise", louder in his left ear, which he attributed to a "surveillance device", and derogatory "whispering voices" present only on the left side. During a previous admission the whistling had been accompanied by a single male voice As his mood improved, the voices faded and disappeared, leaving only a distant whistling in his left ear. Physical examination showed a marked sensorineural deafness in his left ear. He failed to attend for audiometry after discharge. All other investigations were normal.

(ii) A 47-year-old woman had initially been seen by psychiatrists at the age of 18 years because of a sensitive, inadequate personality. Alcohol dependence developed, complicated by grand mal epilepsy. She first complained of auditory hallucinations at the age of 33 years. During subsequent admissions, passivity phenomena, thought insertion, and broadcasting were documented at various times. Mental state examination during the most recent admission revealed a woman of low average intelligence, with slight blunting of affect. She described a persistent buzzing and a solitary, adolescent, female voice, both audible only in her left ear. Four EEGs over a period of 21 years showed diffuse slowing and bilateral spike activity with photic stimulation, most marked over the temporal leads. Audiometry showed bilateral high tone deafness; other investigations were normal.

In both these cases, unilateral hallucinations were present with hearing loss and ipsilateral tinnitus. In the first case the phenomenon appears to represent auditory hyperaesthesia during a depressive episode, leading to functional hallucinations (Hamilton, 1974). In the second case there was additional evidence of non-focal epileptiform activity which may have contributed to both the psychosis and the hallucinations.

ANDREW MCBRIDE
LAWRENCE HAMILTON-KIRKWOOD
$\begin{aligned} & \text { St Cadoc's Hospital } \\ & \text { Caerleon } \\ & \text { Gwent }\end{aligned}$
Reference
HAmitron, M. (1974) Fish's Clinical Psychopathology. Bristol:
Wright.

\section{Inadequate Seizures with Electroconvulsive Therapy}

SIR: Sharpe \& Andrew recently published a Brief Report (Journal, January 1988, 152, 134-136) citing the case of a severely depressed patient in whom treatment with electroconvulsive therapy was abandoned because of repeated failure to induce a convulsion. The authors suggested that failure to convulse may be an occasional and unrecognised cause of non-response to ECT, and concluded that adequate monitoring of seizure activity is required. 